\title{
Self-Adapting Payoff Matrices in Repeated Interactions
}

\author{
Siang Y. Chong and Xin Yao \\ The Centre of Excellence for Research in \\ Computational Intelligence and Applications \\ School of Computer Science \\ University of Birmingham, UK \\ S.Y.Chong;X.Yao@cs.bham.ac.uk
}

\begin{abstract}
Traditional iterated prisoner's dilemma (IPD) assumed a fixed payoff matrix for all players, which may not be realistic because not all players are the same in the real-world. This paper introduces a novel co-evolutionary framework where each strategy has its own self-adaptive payoff matrix. This framework is generic to any simultaneous two-player repeated encounter game. Here, each strategy has a set of behavioral responses based on previous moves, and an adaptable payoff matrix based on reinforcement feedback from game interactions that is specified by update rules. We study how different update rules affect the adaptation of initially random payoff matrices, and how this adaptation in turn affects the learning of strategy behaviors.
\end{abstract}

Keywords: Evolutionary games, Co-evolution, Iterated Prisoner's Dilemma, Mutualism, Repeated Encounter Games

\section{INTRODUCTION}

The IPD game is well-known as the standard metaphor to explain cooperative behaviors among selfish, unrelated individuals [1]. In its classical form, two players engaged in repeated interactions are given two choices: cooperate and defect. The two players receive their payoffs that depend on the choices that they make during the course of behavioral exchange. The game captures the dilemma of cooperating with unrelated, and ultimately selfish players (i.e., those that seek the highest payoffs) by rewarding the player that exploited its opponents with the highest payoff even though the payoff for mutual cooperation is higher than that for mutual defection.

In the IPD, defection is not always the best choice. For example, Axelrod [2], [3] showed through tournaments of experts-designed strategies that a particular form of cooperative strategy, e.g., tit for tat, which cooperates in the first round, and reciprocates thereafter the choice that the opponent made in the previous round, can be viable. Other studies, such as in [1], [4], have explained cooperation in the IPD game using the idea of reciprocal altruism. In particular, cooperation can arise through the mechanism of direct reciprocity, which requires repeated encounters between individuals, so that cooperation received can be returned. Further studies that use co-evolutionary models [5], [6], [7], [8], [9], [10] stressed that cooperative behaviors can be learned through a process of adaptation based on the mechanism of direct reciprocity that is provided through interactions (game play between strategies).

However, all of these studies assumed that the payoff matrix, which defines the reward for making a certain choice in light of the opponent's, is fixed and symmetric. That is, the utility expectation of a strategy on rewards for certain behaviors does not change (i.e., fixed payoff matrix) and is similar for all strategies (i.e., symmetric payoff matrix). These two basic assumptions might not be realistic if the IPD game is used as a model to explain outcomes of real-world interactions due to variations between individuals on the payoff matrix [11]. More importantly, these two assumptions have significant implications to modelling behavioral interactions because they restrict strategies from adapting (e.g., learning) their individual payoff matrices based on feedback of game interactions that reinforces certain behaviors. As such, cooperative outcomes may not be a result of IPD-like payoffs [11]. Instead, cooperative outcomes may be due to a different payoff matrix that favors mutual cooperation (i.e., mutualism [12]).

Here, we present a preliminary study that is aimed at addressing the problem of restrictive assumptions on the payoff matrix (fixed and symmetric) to the evolutionary outcome of repeated encounter games. For this paper, we consider the evolution of strategy payoff matrices through an adaptation process based on reinforcement feedback from behavioral interactions using a fixed update rule, and how this process in turn affects the learning of strategy behaviors. Although this update-rule-based feedback mechanism may not reflect actual mechanisms found in real-world interactions, its simplicity allows for an in-depth study using a co-evolutionary approach to emphasize how the simultaneous adaptations on behavioral responses and the expectations of rewards for behavioral responses affect the learning of behaviors. This approach is different compared to previous studies that consider a fixed, symmetric payoff matrix throughout the evolutionary process [5], [6], [7], [8], [9], [10], or perturbed payoff matrices based on a noise sources with a fixed probability distribution [11], i.e., no learning process on payoff matrices.

Through an empirical study, we show how different update rules, which specify how different elements in the strategy payoff matrices can be reinforced based on past interactions, can affect the adaptation of initially random payoff matrices, which in turn, affects the learning of strategy behaviors for future interactions. On the one hand, when IPD-like update rules are used (i.e., favors exploitation of opponent payoff even though the reinforcement for mutual cooperation payoff is higher than that of mutual defection), defection outcomes are more likely to be obtained. On the ther hand, 
when update rules favor mutual cooperation, cooperative outcomes can be easily obtained. However, update rules only significantly impact the learning of behaviors if the strategy payoff matrix is not overly constrained, e.g., allows for large variations between elements of the strategy payoff matrix from reinforcement feedbacks of game interactions.

The rest of the paper is organized as follows. Section II describes the general setting for iterated, two-player, two-choice games. Section III describes the co-evolutionary model used for the experiments. Section IV presents the results of the experiments, while section $\mathrm{V}$ discusses observations obtained from the experimental results. Finally, section VI concludes the paper, with some remarks for future studies.

\section{ITERATED GAMES WITH TWO CHOICES}

Behavioral interactions can be modelled using a game. One simple example is to use a symmetric, two-player, twochoice game as a model for cooperation [13]. Here, the game consists of two players, each having the choice of either to cooperate or defect. Depending of the choices that both players have made, each player receives a payoff that is specified by a predefined payoff matrix (e.g., Fig. 1). Referring to figure 1, both players receive $R$ units if both cooperates, or $P$ units if both defects. However, if one player cooperates while the other defects, the cooperator will receive $S$ units while the defector receives $T$ units. The values $R$, $S, T$, and $P$ must satisfy the constraints, $R>P$ and $R>(S+T) / 2$. The game is symmetrical if the payoff matrix is the same for both players [13].

\begin{tabular}{|c|lr|rr|}
\hline & \multicolumn{2}{|c|}{ Cooperate } & \multicolumn{2}{|c|}{ Defect } \\
\hline Cooperate & & $\mathrm{R}$ & & $\mathrm{T}$ \\
& $\mathrm{R}$ & $\mathrm{S}$ & & $\mathrm{P}$ \\
\hline Defect & & & & \\
& $\mathrm{T}$ & & $\mathrm{P}$ & \\
\hline
\end{tabular}

Fig. 1. The payoff matrix framework of a two-player, two-choice game. The payoff given in the lower left-hand corner is assigned to the player (row) choosing the move, while that of the upper right-hand corner is assigned to the opponent (column).

Depending on the specifications for $R, S, T$, and $P$, games with different characteristics can be produced. Considering a single iteration, when $T>R>P>S$, one obtains the prisoner's dilemma game, where the best choice is to defect. However, when $R>T$ and $S>P$, one obtains the mutualism game, where the best choice is to cooperate [12]. Both of these games can be extended to have more than one iteration, i.e., iterated (repeated encounter) games. Figures 2 and 3 give examples of payoff matrices for the IPD and iterated mutualism games, respectively [11].

For this paper, we propose a generic framework of repeated encounter games, whereby the payoff matrix is different and adaptable for each strategy. That is, the framework is

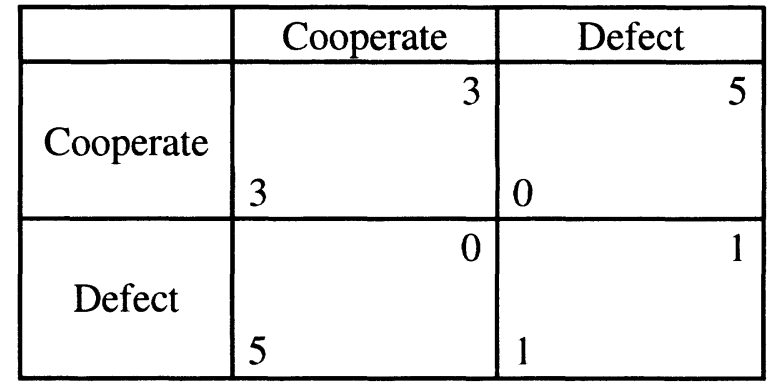

Fig. 2. Typical payoff matrix for an IPD game. The payoff given in the lower left-hand corner is assigned to the player (row) choosing the move, while that of the upper right-hand corner is assigned to the opponent (column).

\begin{tabular}{|c|lr|rr|}
\hline & \multicolumn{2}{|c|}{ Cooperate } & \multicolumn{2}{c|}{ Defect } \\
\hline Cooperate & & 5 & & \\
& 5 & 1 & & \\
\hline Defect & & & & \\
& 3 & & 0 \\
\hline
\end{tabular}

Fig. 3. Typical payoff matrix for an iterated mutualism game. The payoff given in the lower left-hand corner is assigned to the player (row) choosing the move, while that of the upper right-hand corner is assigned to the opponent (column).

applicable to any repeated encounter game with different payoff matrices, and not just limited to those that satisfy specific constraints (e.g., IPD or mutualism games).

\section{Co-EVolutionary Models For LEARNing PAYOFF MATRIX}

\section{A. Strategy Representation}

We limit our investigation to only deterministic, memoryone strategy, both for simplicity and a starting point for a study on the effect of strategies with different, but adaptable payoff matrices. As such, we require a simple strategy representation that consists of: (a) a behavioral response part (e.g., determines the choice to make based on choices made in the previous move), and, (b) a payoff matrix part (e.g., determines what payoff the strategy should receive). To achieve this, we use the simple direct look-up table representation [14], which allows direct behavioral evolution of strategies, to represent a strategy's behavioral responses based on previous moves. The look-up table is extended to store values of a two-choice payoff matrix.

Figure 4 illustrates the direct look-up table representation for the strategies with two choices and memory-one. $m_{i j}, i, j=1,2$ specifies the choice to be made, given the inputs $i$ (player's own previous choice) and $j$ (opponent's previous choice). Rather than using pre-game inputs (two for memory-one strategies), the first move is specified independently, $m_{f m}$. Binary choices of +1 and -1 are used. The payoff matrix part for each strategy specifies payoffs 
that the strategy receives given choices made by the strategy and its opponent for that particular iteration. The elements in the payoff matrix, $p_{i j}, i, j=1,2$, are not fixed, but instead, variable and depends on choices made by the strategy during interactions. That is, evolutionary variation is not applied directly to the payoff matrix (details of the update rule used to adapt $p_{i j}$ is presented in the following section).

\begin{tabular}{cc|cc} 
& & \multicolumn{2}{|c}{ Opponent's Previous Move } \\
& & +1 & -1 \\
\hline Player's & +1 & $m_{11}$ & $m_{12}$ \\
Previous Move & -1 & $m_{21}$ & $m_{22}$
\end{tabular}

(a) Behavior

\begin{tabular}{cc|cc} 
& & \multicolumn{2}{|c}{ Opponent's Previous Move } \\
& & +1 & -1 \\
\hline Player's & +1 & $p_{11}$ & $p_{12}$ \\
Previous Move & -1 & $p_{21}$ & $p_{22}$
\end{tabular}

(b) Payoff Matrix

Fig. 4. The look-up table representation for the two-player repeated encounter game with two choices and memory length one. (a) Behavioral Response (also includes $m_{f m}$ for the first move, which is not shown in the figure). (b) Payoff Matrix (utility expectation).

A simple mutation operator is used to generate offspring from parents. When a response, i.e., the element of the direct look-up table for behavioral responses (includes $m_{f m}$ and $m_{i j}$ ) mutates, it changes to the other possible choice. Each table element has a fixed probability, $p_{m}$, of being replaced by the other choice. The value $p_{m}$ is not optimized. In effect, the variation operator for the strategy representation of the two-choice IPD used here is a uniform mutation with probability $p_{m}$.

Crossover is not used in any experiment. With the direct look-up table for strategy representation, any variation operator will introduce variations on behavioral responses directly. As investigated earlier in [14] (even for the more complex IPD game with intermediate choices), a simple mutation operator is more than sufficient to introduce the required variations of strategy behaviors. The use of crossover is not necessary.

\section{B. Co-evolutionary Procedure}

The co-evolutionary learning approach that is used here is similar to that used in [14]. The original system in [14] is changed to accommodate the co-evolutionary learning of strategy behavioral responses and payoff matrix. Given that the adaptation process occurs for strategy payoff matrix as well, more experiments are conducted to investigate the impact of initial strategy payoff matrices on the learning of strategy behaviors. The initialization schemes are summarized in table $\mathrm{I}$.

The following gives the flow of the co-evolutionary procedure:

\section{TABLE I}

INITIALIZATION SCHEMES FOR STRATEGY PAYOFF MATRIX OF INITIAL POPULATION. $U(0,5)$ REFERS TO A RANDOM VALUE DRAWN FROM A UNIFORM DISTRIBUTION BETWEEN 0 AND 5. ASYM REFERS TO THE INITIALIZATION SCHEME WHERE STRATEGIES CAN BE INITIALIZED

WITH DIFFERENT PAYOFF MATRICES. SYM REFERS TO THE

INITIALIZATION SCHEME WHERE STRATEGIES ARE INITIALIZED WITH THE SAME PAYOFF MATRIX.

\begin{tabular}{|c|c|c|c|c|c|c|}
\hline & RanAll & Ran & IPD1 & IPD2 & MUT1 & MUT2 \\
\hline$p_{11}$ & $U(0,5)$ & $U(0,5)$ & 4 & 3 & 5 & 5 \\
\hline$p_{12}$ & $U(0,5)$ & $U(0,5)$ & 0 & 0 & 1 & 1 \\
\hline$p_{21}$ & $U(0,5)$ & $U(0,5)$ & 5 & 5 & 4 & 3 \\
\hline$p_{22}$ & $U(0,5)$ & $U(0,5)$ & 1 & 1 & 0 & 0 \\
\hline & ASYM & SYM & SYM & SYM & SYM & SYM \\
\hline
\end{tabular}

1) Generation step, $t=0$ : Initialize $N / 2$ parent strategies, $P_{i}, i=1,2, \ldots, N / 2$, randomly.

a) Each table element, $m_{f m}$ and $m_{i j}, i, j=1,2$, is initialized to values, +1 or -1 , each with equal probability.

b) Each element for the payoff matrix, $p_{i j}, i, j=$ 1,2 , is initialized according to values depending on the investigated initialization scheme (table I).

2) Generate $N / 2$ offspring, $O_{i}, i=1,2, \ldots, N / 2$, from $N / 2$ parents using a mutation operator with probability $p_{m}$

3) All pairs of strategies compete, including the pair where a strategy plays itself (i.e., round-robin tournament). For $N$ strategies in a population, every strategy competes a total of $N$ games. Each strategy's payoff matrix is updated after every iteration for each game. The updated payoff matrix is used immediately in the next iteration. The updates are described as follows:

a) Player cooperates, opponent cooperates:

$p_{11}^{\prime}=p_{11}+\delta_{11}$

b) Player defects, opponent cooperates:

$p_{21}^{\prime}=p_{21}+\delta_{21}$

c) Player cooperates, opponent defects:

$p_{12}^{\prime}=p_{12}+\delta_{12}$

d) Player defects, opponent defects:

$p_{22}^{\prime}=p_{22}+\delta_{22}$

where $\delta_{i j} \geq 0, i, j=1,2$.

4) Select the best $N / 2$ strategies based on total payoffs of all games played. Increment generation step, $t=t+1$.

5) Step 2 to 4 are repeated until termination criterion (i.e., a fixed number of generation) is met.

In particular, we use $N=20$, and repeat the coevolutionary process for 200 generations, which is sufficiently long to observe an evolutionary outcome (e.g., persistent cooperation). A fixed game length of 150 iterations is used for all games. Experiments are repeated for 30 independent runs.

The update rule, i.e., $\delta_{i j}, i, j=1,2$, determines how the elements for each strategy's payoff matrix part are changed based on the strategy's interactions with the opponent. The 
update rule follows a reinforcement view whereby only the element in the payoff matrix that corresponds to the pair of choices made by the strategy and its opponent is updated. Note that the adaptation process of the strategy's payoff matrix is indirect, i.e., evolutionary variation is not applied directly to the payoff matrix. Instead, variation to the payoff matrix is a result of interactions between strategies, which depend on their behavioral responses that are subjected to evolutionary variations. Lastly, for simplicity, we assume that the update rule (e.g., given by the $\delta \mathrm{s}$ ) is the same for all strategies.

As such, it is emphasized that not all interactions conform to a specific payoff matrix (e.g., IPD) because each strategy's payoff matrix is evolving as well. With this in mind, we can investigate what payoff matrices that emerged, and also what behaviors that are obtained based on those payoff matrices from a co-evolutionary process.

\section{RESULTS}

\section{A. Learning Behaviors when Exploitation of Opponents is Favored}

We first consider IPD-like update rules to investigate how update rules can affect the evolution of payoff matrices, and in turn, the learning of strategy behaviors. IPD-like update rules favor exploitation of opponents, even though reinforcement for mutual cooperation is higher compared to that of mutual defection. That is, the values for $\delta \mathrm{s}$ in the update rule satisfy the IPD constraints, i.e., $\delta_{21}>\delta_{11}>$ $\delta_{22}>\delta_{12}$. The following experiments are motivated to facilitate the investigation on what strategy payoff matrices and behaviors that emerged when the update rule favors strategies exploiting their opponents.

Table II summarizes and compares all update rules used for the experiments in this paper. We first focus on the $U R_{I P D 1}$ update rule with the following $\delta$ values, $\delta_{11}=0.4 / 150$, $\delta_{21}=0.5 / 150, \delta_{12}=0.0 / 150, \delta_{22}=0.1 / 150$. Given that an update to a strategy's payoff matrix is made after every iteration, we scale the $\delta$ s to the number of iteration of a game (e.g., dividing values by 150 ).

TABLE II

UPDATE RULES USED FOR THE EMPIRICAL STUDY. BOTH $U R_{I P D 1}$ AND $U R_{I P D 2}$ UPDATE RULES FAVOR EXPLOITATION OF OPPONENTS. WITH

$U R_{I P D 2}$, THE REINFORCEMENT OF REWARDS FOR EXPLOITATION OF OPPONENTS IS FURTHER EMPHASIZED. $U R_{M U T}$ UPDATE RULE FAVORS MUTUAL COOPERATION

\begin{tabular}{|c|c|c|c|}
\hline & $U R_{I P D 1}$ & $U R_{I P D 2}$ & $U R_{M U T}$ \\
\hline$\delta_{11}$ & $0.4 / 150$ & $0.4 / 150$ & $0.5 / 150$ \\
\hline$\delta_{12}$ & $0.0 / 150$ & $0.0 / 150$ & $0.1 / 150$ \\
\hline$\delta_{21}$ & $0.5 / 150$ & $0.7 / 150$ & $0.3 / 150$ \\
\hline$\delta_{22}$ & $0.1 / 150$ & $0.1 / 150$ & $0.0 / 150$ \\
\hline
\end{tabular}

For the first experiment, we considered the RanAll initializations of strategy payoff matrices (table I). Results show the populations of 12 runs out of 30 evolved to mutual cooperation (RanAll row and $U R_{I P D 1}$ column in table III). Closer inspection shows that surviving strategies converged to a similar payoff matrix, and that the the element in the payoff matrix corresponding to mutual cooperation is much higher compared to the other elements in these 12 mutual cooperative runs (e.g., $p_{11} \gg p_{12}, p_{21}, p_{22}$ ). This observation suggests strategies evolving to cooperative behaviors early on after a random initialization. The persistent cooperation to the end of the run is a result of continued reinforcement feedback between mutual cooperative play and strategies' expectations on rewards for mutual cooperation.

The populations in the remaining 18 runs evolved to a point where they engaged in mutual defection. Similar observation of strategies converging to a similar payoff matrix is made. Closer inspection reveals that the element in the payoff matrix corresponding to mutual defection is much higher than other elements except that of the element corresponding to exploitation of opponents (or temptation to defect). That is, $p_{22} \gg p_{11}, p_{12}$ and $p_{22}>p_{21}$, suggesting that the defection outcomes are the result of initial random strategies evolving to exploit one another first, before evolving to play mutual defection.

In general, results from the experiment (RanAll) suggest that cooperative outcomes are less likely to be obtained when the IPD-like update rule, which favors strategies exploiting their opponents, is used. This is also observed from results summarized in table IV that shows the average cooperation frequency of 30 runs for each experiment. In particular, in the RanAll row and $U R_{I P D 1}$ column of table IV, the average cooperation frequency of $40.06 \%$ (less than $50 \%$ ) shows that the co-evolutionary process resulted with more defection plays.

However, given the convergence to a similar payoff matrix for the co-evolving population, we conducted additional experiments with different initializations of payoff matrices (table I) to determine their impact on the evolutionary outcome. Results of these experiments are summarized in $U R_{I P D 1}$ column of tables III, IV, and V. In particular, no statistically significant difference is observed for comparison between results of experiments ( $U R_{I P D 1}$ column of table $\mathrm{V}$ ) where all strategies were initialized with the same random payoff matrix (Ran) and where each strategy was randomly initialized with different payoff matrix (RanAll). Similarly, no statistically significant difference is observed for conparison between the RanAll experiment and experiments where strategy payoff matrices were initialized with the same IPD payoff matrix (IPD1 and IPD2 in table I). However, when strategy payoff matrices were initialized with mutualism-like payoffs (MUT1 and MUT2 in table I), cooperative outcomes were obtained easily, e.g., the higher number of cooperative outcomes $\left(U R_{I P D 1}\right.$ column of table III) and higher average cooperation frequencies $\left(U R_{I P D 1}\right.$ column of table IV) are statistically significantly different compared to that of RanAll experiment.

Furthermore, as in the original experiment (RanAll), strategies are also observed to converge to a similar payoff matrix regardless of how strategy payoff matrices are initialized. In particular, runs with cooperative outcome are always a 
result of strategies converging to a similar payoff matrix with a very high value for the element corresponding to mutual cooperation. On the other hand, runs with defection outcome are always a result of strategies converging to a similar payoff matrix with a very high value for the element corresponding to mutual defection. However, given that the update rule remained fixed in these experiments, these results suggest that the initial payoff matrices of strategies can affect the outcome of the co-evolutionary process since strategy behavioral responses are randomly initialized.

In addition, we conducted further experiments whereby the reinforcement value in the update rule corresponding to exploitation of opponents (i.e., $\delta_{21}$ ) is further increased with respect to other values to further clarify the impact of IPDlike update rules on the evolution of strategy payoff matrices and behaviors. We consider the $U R_{I P D 2}$ update rule (table I), where value for $\delta_{21}$ in the update rule is increased from $0.5 / 150$ to $0.7 / 150$ (note that since $\delta_{11}=0.4 / 150$ and $\delta_{22}=0.0 / 150$, then $\delta_{21}<0.8 / 150$ to obtain IPD-like update rules). Comparison of results in $U R_{I P D 2}$ column with $U R_{I P D 1}$ column of tables III and IV shows that both the number of runs with cooperative outcomes and the average cooperation frequency are reduced when the $U R_{I P D 2}$ update rule was used, regardless of how strategy payoff matrices were initialized. With the exception of RanAll experiments, comparison of the pairs of experiments with different update rules but similar initializations of strategy payoff matrices indicates statistical significant differences $\left(U R_{I P D 2}\right.$ column of table V). These results suggest that update rules that favor exploitation of opponents lead to evolution of mutual defection play.

\section{B. Learning Behaviors when Mutual Cooperation is Favored}

Earlier, we investigated IPD-like update rules that favor strategies exploiting their opponents even though reinforcement for mutual cooperation is higher compared to that of mutual defection. However, other update rules can also be investigated, depending on how update rules are interpreted to favor specific behaviors. Here, we investigate the impact of update rules that favor mutual cooperation, e.g., mutualismlike update rules: $\delta_{11}=0.5 / 150, \delta_{21}=0.3 / 150, \delta_{12}=$ $0.1 / 150, \delta_{22}=0.0 / 150\left(U R_{M U T}\right.$ in table I). $\delta$ s are scaled from values used in [11] to the number of iteration used. Note that any combination of $\delta \mathrm{s}$ that satisfy mutualism constraints can be used, i.e., the update rule favors mutual cooperation.

Results of experiments are summarized in column $U R_{M U T}$ of tables III, IV, and V. They show that regardless of how strategy payoff matrices are initialized, cooperative outcomes are always obtained. That is, populations in all 30 runs of all experiments evolved to mutual cooperation play (column $U R_{M U T}$ of tables III). Further inspection shows that in most runs, strategies converge to a mutualism-like payoff matrix, with the element corresponding to mutual cooperation (e.g., $p_{11}$ ) having a value that is significantly higher compared to other elements. That is, strategies learn cooperative behaviors and mutualism-like payoff matrices. This observation is consistent for other experiments with different initialization of payoff matrices. This suggests that cooperation can be learned easily if update rules that favor mutual cooperation are used.

\section{Learning Behaviors when Reinforcement Feedbacks to Expectations of Rewards are Constrained}

All experiments conducted thus far allowed for strategy payoff matrices to be updated indefinitely without any constraint. That is, the value for an element with positive reinforcements gets larger as it is updated. When this reinforcement process is allowed to continue long enough, there is no longer any trade-off in reinforcing other elements in the strategy payoff matrix, resulting with convergence to a specific behavioral response (e.g., mutual cooperation or mutual defection) because the particular element in the strategy payoff matrix corresponding to that response is much larger compared to the other elements. At this point, further evolution will only result with this reinforcement feedback loop between the converged behavioral response and its corresponding expectation of reward.

As such, we investigate the impact of constrained strategy payoff matrix on the evolutionary outcome. We consider a simple a constraint, i.e., $\sum_{i, j}^{2} p_{i j} \leq C$ (where $C$ is a predetermined and fixed constant) that is applied to all strategies. Any particular update on a $p_{i j}$ that results with $\sum_{i, j}^{2} p_{i j}>C$ is discarded, i.e., updated $p_{i j}^{\prime}$ is the same as original $p_{i j}$. We first consider the impact of this constrained strategy payoff matrix to the evolutionary outcome for the experiment with $U R_{I P D 1}$ update rule and RanAll initialization. The following $C$ values are considered: $10,12,14,16,18,20,30,40,50$, $60,70,80$, and, 90 .

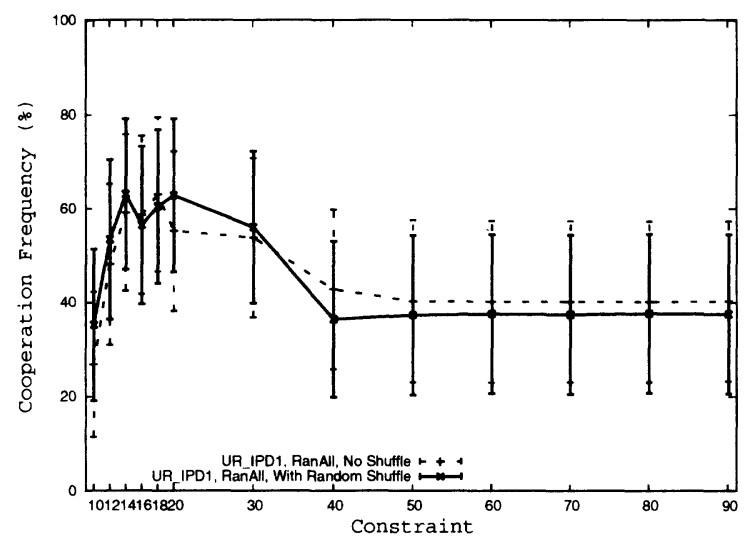

Fig. 5. Results of experiments with $U R_{I P D 1}$ update rule and RanAll initialization with constrained strategy payoff matrix. The first graph (dashed line) plots the average cooperation frequency in \% of 30 runs (taken at the end of the run) with 95\% confidence interval as the value for $C$ is changed. The second graph (bold line) plots the result when strategies are randomly shuffled at the start of each generation before competitions.

Figure 5 plots the average cooperation frequency $(\%)$ of all 30 runs taken at the end of the co-evolutionary run. The graph with dashed line shows that average cooperation frequencies are greater than $50 \%$ (e.g., more than half the total number of runs resulted with mutual cooperation) for $C$ values between 14 and 30, and less than 50\% (e.g., more than half the total 
TABLE III

RESULTS OF EXPERIMENTS WITH DIFFERENT UPDATE RULES FOR STRATEGY PAYOFF MATRIX. ALL RESULTS ARE TAKEN AT THE END OF CO-EVOLUTIONARY RUN. "No < 25\%” INDICATES THE NUMBER OF RUNS WHERE THE MAJORITY PLAY IS DEFECTION. "No $>75 \%$ ” INDICATES THE NUMBER OF RUNS WHERE THE MAJORITY OF PLAY IS COOPERATION.

\begin{tabular}{|c|c|c||c|c||c|c|}
\hline & \multicolumn{2}{|c||}{$U R_{I P D 1}$} & \multicolumn{2}{c||}{$U R_{I P D 2}$} & \multicolumn{2}{c|}{$U R_{M U T}$} \\
\cline { 2 - 7 } & $N o<25 \%$ & $N o>75 \%$ & $N o<25 \%$ & No $>75 \%$ & No $<25 \%$ & No $>75 \%$ \\
\hline RanAl1 & 18 & 12 & 20 & 10 & 0 & 30 \\
\hline Ran & 13 & 17 & 22 & 8 & 0 & 30 \\
\hline IPD1 & 16 & 14 & 22 & 8 & 0 & 30 \\
\hline IPD2 & 17 & 13 & 24 & 6 & 0 & 30 \\
\hline MUT1 & 7 & 21 & 16 & 14 & 0 & 30 \\
\hline MUT2 & 5 & 25 & 16 & 14 & 0 & 30 \\
\hline
\end{tabular}

TABLE IV

RESULTS OF EXPERIMENTS WITH DIFFERENT UPDATE RULES FOR STRATEGY'S PAYOFF MATRIX. ALL RESULTS ARE TAKEN AT THE END OF CO-EVOLUTIONARY RUN. RESULTS ARE THE AVERAGE OF COOPERATION FREQUENCY OVER 30 RUNS IN \% WITH A CONFIDENCE INTERVAL OF 95\% IN \% AS WELL.

\begin{tabular}{|c|c|c|c|}
\hline & $U R_{I P D 1}$ & $U R_{I P D 2}$ & $U R_{M U T}$ \\
\hline RanAll & $40.06 \pm 17.28$ & $33.86 \pm 16.60$ & $97.32 \pm 0.80$ \\
Ran & $56.22 \pm 17.41$ & $27.76 \pm 15.65$ & $97.24 \pm 1.30$ \\
IPD1 & $47.51 \pm 17.70$ & $28.31 \pm 15.66$ & $97.99 \pm 0.96$ \\
IPD2 & $43.89 \pm 17.74$ & $21.29 \pm 13.97$ & $98.15 \pm 0.78$ \\
MUT1 & $69.87 \pm 16.12$ & $47.54 \pm 17.59$ & $97.74 \pm 1.29$ \\
MUT2 & $82.42 \pm 13.03$ & $48.21 \pm 17.59$ & $97.77 \pm 1.22$ \\
\hline
\end{tabular}

TABLE V

COMPARISON OF EXPERIMENTS USING A $t$-TEST FOR STATISTICALLY SIGNIFICANT DIFFERENCE AT A 0.05 LEVEL OF SIGNIFICANCE BY A TWO-TAILED TEST WITH 29 DEGREE OF FREEDOM. EACH TABLE ELEMENT CONSISTS OF RESULTS FROM TWO $t$-TESTS (SEPARATED BY A COMMA) THAT COMPARE THE AVERAGE COOPERATION FREQUENCIES OF EXPERIMENTS: (1) TEST RESULTS BETWEEN RANALL AND OTHERS (WITH THE SAME

UPDATE RULE), (2) TEST RESULTS BETWEEN $U R_{I P D 1}$ AND OTHERS (WITH THE SAME INITIALIZATIONS OF STRATEGY PAYOFF MATRIX). “-” INDICATES THAT A TEST IS NOT POSSIBLE. VALUES WITH “†” INDICATES A STATISTICAL SIGNIFICANT DIFFERENCE.

\begin{tabular}{|c|c|c|c|}
\hline & $U R_{I P D 1}$ & $U R_{I P D 2}$ & $U R_{M U T}$ \\
\hline RanAll &,-- &,- 1.42 &,$--6.59^{\dagger}$ \\
Ran & $-1.45,-$ & $0.61,3.47^{\dagger}$ & $0.11,-4.69^{\dagger}$ \\
IPD1 & $0.83,-$ & $0.71,2.31^{\dagger}$ & $-1.09,-5.73^{\dagger}$ \\
IPD2 & $0.44,-$ & $1.43,3.00^{\dagger}$ & $-1.38,-6.11^{\dagger}$ \\
MUT1 & $-2.93^{\dagger},-$ & $-1.55,2.63^{\dagger}$ & $-0.61,-3.45^{\dagger}$ \\
MUT2 & $-4.37^{\dagger},-$ & $-1.64,4.07^{\dagger}$ & $-0.67,-2.33^{\dagger}$ \\
\hline
\end{tabular}

number of runs resulted with mutual defection) for other $C$ values. In particular, average cooperation frequencies stay around $40 \%$ (similar to the experiment without constraint to updating the strategy payoff matrix, e.g., RanAll row and $U R_{I P D 1}$ column of table IV) starting around $C$ value of 40 .

Note that similar observation is obtained when the experiments are repeated with random shuffling of strategies at the start of each generation (graph plotted with bold line in Fig. 5), i.e., there are no statistical significant differences between the two experiment sets within $95 \%$ confidence interval for different $C$ values. This is also true for all other experiments in this study (results omitted to save space). In general, given the small population of competing strategies (e.g., 20), the evolutionary outcome does not depend on the order of strategy encounters.
However, different results were obtained when different updates rules were used. For example, figure 6 shows the results of plots when $U R_{I P D 1}$ (dashed line), $U R_{I P D 2}$ (bold line), and $U R_{M U T}$ (thin line) update rules were used. On the one hand, comparison of experiments that used $U R_{I P D 2}$ and $U R_{I P D 1}$ shows that the graph for $U R_{I P D 2}$ results with lower average cooperation frequencies for most of $C$ values considered. On the other hand, comparison of experiments that used $U R_{M U T}$ and $U R_{I P D 1}$ shows that the graph for $U R_{M U T}$ results with higher average cooperation frequencies for most of $C$ values considered. As with the experiment that used $U R_{I P D 1}$, average cooperation frequencies for the experiments that used $U R_{I P D 2}$ and $U R_{M U T}$ converged to their respective values that correspond to the experiment without constraint starting around $C$ value of 40 . 


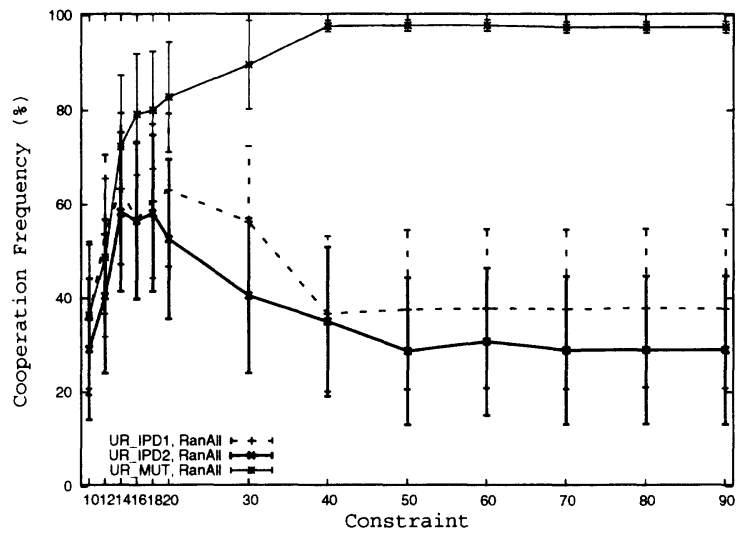

Fig. 6. Results of experiments with different update rules and RanAll initialization with constrained strategy payoff matrix. Strategies are randomly shuffled at the start of each generation for all experiments. All graphs plot the average cooperation frequency in \% of 30 runs (taken at the end of the run) with $95 \%$ confidence interval as the value for $C$ is changed. The graphs are plotted using dashed, bold, and thin lines for experiments with $U R_{I P D 1}, U R_{I P D 2}$, and $U R_{M U T}$ update rules, respectively.

Results from figure 6 further suggest that update rules that favor expectation of rewards for different behavioral responses affect the co-evolutionary learning of certain behaviors. In general, when constraint for updating payoff matrix is relaxed (i.e., higher $C$ values), the impact of update rules is more pronounced. For example, IPD-like update rules will result with more mutual defection due to having higher expectation of rewards for mutual defection. With mutualism-like update rules, the opposite occurs, i.e., mutual cooperation is expected.

However, when constraint for updating payoff matrix is tightened (i.e., lower $C$ values), the impact of update rules is less pronounced. In the case of IPD-like update rules, mutual cooperation is possible and more likely. For example, figure 6 shows that average cooperation frequencies are higher than $50 \%$ between $C$ values of 14 and 20 for both IPD-like update rules (e.g., $U R_{I P D 1}$ and $U R_{I P D 2}$ ).

This observation can be explained by considering that the constraint determines the amount of variations between the four elements of the strategy payoff matrix due to reinforcement feedbacks from game interactions. With a relaxed constraint, i.e., higher $C$ values, variations between the elements tend to be larger due to the reinforcement feedbacks that favor a particular response. With a tighter constraint, i.e., lower $C$ values, variations between the elements are smaller. For the case of IPD-like update rules, setting a higher $C$ value allows the update rule to first reinforce exploitation of opponents before before reinforcing mutual defection play. However, when a low $C$ value is used, mutual cooperation play is possible given that the co-evolutionary system with direct look-up table for strategy representation evolves early on to mutual cooperation (full discussion is presented in [14]). The update rule has no impact on the evolutionary process because the constraint disallows further reinforcement to elements of strategy payoff matrix. That is, the co-evolutionary learning system behaves as though there is no adaptation of strategy payoff matrix.

\section{Discussion}

A. Learning Strategy Behaviors with Fixed Payoff Matrix is Not the Same as Learning Strategy Behaviors with Different Payoff Matrices

Most of the previous studies on evolving strategies using co-evolution [5], [6], [7], [8], [9], [10], [14] assumed a fixed, symmetric payoff matrix. Behaviors are learned through a process of adaptation on a strategy representation, with the payoff-based fitness determined using a pre-defined global payoff matrix. Evolutionary pressure is thus exerted only on strategy behavioral responses. As such, strategies learned certain behaviors based on the outcome of the evolutionary process.

This is different compared to the co-evolutionary learning process considered here, where strategies can have different payoff matrices that are adaptable through a reinforcement feedback process (using update rules) that is based on behavioral interactions. When strategies start with both random behavioral responses and payoff matrices, evolutionary pressure is exerted not only on behavioral responses, but also the payoff matrices of strategies because both are responsible for determining their fitnesses. Here, strategies not only learn behaviors, but also the utility expectations that determine how behaviors are rewarded (i.e., payoff matrix).

\section{B. Different Update Rules Lead to Different Evolutionary Outcomes}

Results from experiments have shown that different update rules lead to different evolutionary outcomes. Starting with random initialization of strategy behaviors, defection outcomes are more likely to be obtained if IPD-like update rules that favor exploitation of opponents are used. However, the evolutionary outcome (defection) is also dependent on how strategy payoff matrices are initialized, and how the reinforcement value corresponding to exploitation of opponents are defined.

These two factors can be explained by considering that the evolved mutual defection play is the best response to earlier evolved strategies that are exploiting one another. At the start of the evolutionary process, strategies that exploit others receive increasingly higher payoffs compared to other plays due to the update rule reinforcing the element corresponding to exploitation of opponents in the strategy payoff matrix with higher proportions. After that, given that the element corresponding to being exploited is not reinforced (i.e., $d_{12}=$ 0 ), strategies eventually evolve to play defection only because the lost of fitness for being exploited is too large for strategies to consider playing cooperation.

However, when the update rule is changed so that it now favors mutual cooperation play, results from experiments show that persistent mutual cooperation can be easily evolved, regardless of how strategy payoff matrices are initialized. That is, even when strategy payoff matrices are initialized so that the element corresponding to exploitation 
of opponents are higher compared to the others (e.g., IPD payoff matrices), strategies that engaged in mutual cooperation receives increasingly higher payoffs due to the update rule reinforcing the element corresponding to mutual cooperation in the strategy payoff matrix with higher proportions.

It should be noted that the impact of update rules on the evolution of behaviors is only significant if there are sufficient reinforcements from game interactions that result with large variations between elements in a strategy payoff matrix. That is, if updates to the strategy payoff matrix are not overly constrained to allow for sufficient variations between the elements that correspond to expectations of rewards for different responses from reinforcement feedbacks of game interactions, update rules with different emphasis on behavioral responses can significantly affect the coevolutionary learning of strategy behaviors.

\section{CONCLUSION}

This paper presents a preliminary study on evolving strategy payoff matrices, and how such an adaptation process can affect the learning of strategy behaviors. The study is motivated from the observation that the assumption of having fixed, symmetric payoff matrix for all evolving strategies may not be realistic. Furthermore, the assumption of fixed, symmetric payoff matrix is highly restrictive because it does not allow strategies adapting their individual payoff matrices based on feedback of game interactions that reinforces certain behaviors.

To facilitate our investigation on the impact of relaxing the restrictive assumption of fixed, symmetric payoff matrix for each strategy, thereby allowing strategies to have different payoff matrices that are also adaptable, we focus specifically on an adaptation process of payoff matrix based on past behavioral interactions. A simple update rule that reinforces the elements of the payoff matrix is considered. The update rule provides a reinforcement feedback process between strategy behaviors and payoff matrices during the co-evolutionary process.

The result is a co-evolutionary process, whereby the evolutionary outcome is dependent on the adaptation process of both behaviors (i.e., strategy behavioral responses) and utility expectations that determine how behaviors are rewarded (i.e., strategy payoff matrices). In particular, experiments are conducted to show how different update rules affect the adaptation process of payoff matrices, which in turn, affect the learning of strategy behaviors. Results show that defection outcomes are more likely to be obtained if IPD-like update rules that favor the exploitation of opponents are used. However, cooperative outcomes can be easily obtained when mutualism-like update rules that favor mutual cooperation are used. Update rules affect the learning of strategy behaviors when they lead to large variations between elements in the strategy payoff matrix (e.g., mutualism-like update rule results with a significantly larger element corresponding to mutual cooperation in the payoff matrix compared to the others).
It is noted that the update-rule-based feedback mechanism may not reflect actual mechanisms in real-world interactions even though its simplicity allows for an in-depth study on how simultaneous adaptations of behavioral responses and expectations on rewards for behavioral responses affect the learning of strategy behaviors. For future work, studies should be carried out to determine how adaptations of behavioral responses and that of the expectations on rewards are linked with each other, and how this link can be abstracted and modelled as a mechanism in the coevolutionary framework introduced here.

\section{ACKNOWLEDGMENTS}

The authors are grateful to EPSRC for its support through Grant GR/S63472/01, to ARC (Australian Research Council) for its support through an ARC International Link grant, and to the School of Computer Science at the University of Birmingham for its studentship to the first author.

\section{REFERENCES}

[1] R. Axelrod, The Evolution of Cooperation. New York: Basic Books, 1984.

[2] — "Effective choice in the prisoner's dilemma," The Journal of Conflict Resolution, vol. 24, no. 1, pp. 3-25, Mar. 1980.

[3] _ - "More effective choice in the prisoner's dilemma," The Journal of Conflict Resolution, vol. 24, no. 3, pp. 379-403, Sept. 1980.

[4] M. A. Nowak and K. Sigmund, "Tit for tat in heterogeneous populations," Nature, vol. 355, pp. 250-253, 1992.

[5] R. Axelrod, "The evolution of strategies in the iterated prisoner's dilemma," in Genetic Algorithms and Simulated Annealing, L. D. Davis, Ed. New York: Morgan Kaufmann, 1987, ch. 3, pp. 32-41.

[6] D. B. Fogel, "The evolution of intelligent decision making in gaming," Cybernetics and Systems: An International Journal, vol. 22, pp. 223236, 1991.

[7] _ "Evolving behaviors in the iterated prisoner's dilemma," Evolutionary Computation, vol. 1, no. 1, pp. 77-97, 1993.

[8] — - "On the relationship between the duration of an encouter and the evolution of cooperation in the iterated prisoner's dilemma," Evolutionary Computation, vol. 3, no. 3, pp. 349-363, 1996.

[9] P. Darwen and X. Yao, "On evolving robust strategies for iterated prisoner's dilemma," in Progress in Evolutionary Computation, ser. Lecture Notes in Artificial Intelligence, vol. 956, 1995, pp. 276-292.

[10] B. A. Julstrom, "Effects of contest length and noise on reciprocal altruism, cooperation, and payoffs in the iterated prisoner's dilemma," in Proc. 7th International Conf. on Genetic Algorithms (ICGA'97). San Francisco, CA: Morgan Kauffman, 1997, pp. 386-392.

[11] D. D. P. Johnson, P. Stopka, and J. Bell, "Individual variation evades the prisoner's dilemma," BMC Evolutionary Biology, vol. 2, no. 15, 2002.

[12] K. C. Clements and D. W. Stephens, "Testing models of non-kin cooperation: Mutualism and the prisoner's dilemma," Animal Behaviour, vol. 50, pp. 527-535, 1995.

[13] M. Mesterton-Gibbons and L. A. Dugatkin, "Cooperation among unrelated individuals: Evolutionary factors," The Quarterly Review of Biology, vol. 67, no. 3, pp. 267-281, 1992.

[14] S. Y. Chong and X. Yao, "Behavioral diversity, choices, and noise in the iterated prisoner's dilemma," IEEE Transactions on Evolutionary Computation, vol. 9, no. 6, pp. 540-551, 2005. 